\title{
HEAT KERNEL SMOOTHING ON UNIT SPHERE
}

\author{
Moo K. Chung \\ Department of Statistics, Biostatistics, and Medical Informatics \\ Waisman laboratory for brain imaging and behavior \\ University of Wisconsin \\ Madison, WI 53706 \\ mchung@stat.wisc.edu
}

\begin{abstract}
In brain imaging, cortical data such as the cortical thickness, cortical surface curvatures and surface coordinates have been mapped to a unit sphere for the purpose of visualization, surface registration and statistical analysis. Since the unit sphere provides a readily available parametrization and basis functions, cortical data can be easily quantified with respect to the spherical parametrization. For the cortical data on the unit sphere, it is necessary to smooth them to increase the signal-to-noise ratio and the smoothness for the subsequent statistical analysis. We present a mathematical framework for smoothing data on a unit sphere using the heat kernel. The heat kernel is analytically constructed using the spherical harmonics and $O(n)$ heat kernel smoothing algorithm is presented.
\end{abstract}

\section{INTRODUCTION}

Due to the highly convoluted nature of the human brain cortex, some sort of normalization onto a parameterized standard surface is necessary for quantifying cortical data. One such normalization technique is the cortical flattening that maps the convoluted cortical manifold into a unit sphere with the minimum geometric distortion $[7,10]$. Alternately, one may flatten the cortex using the inverse process of a deformable surface algorithm, which deforms a sphere to match the cortex [11].

Once the cortical data is mapped onto the unit sphere, spherical harmonics have been used in modeling the data. The major use of spherical harmonics in medical imaging has been in representing the coordinates of closed anatomical boundaries [2, 8, 13]. In this study, we use the spherical harmonics for developing a kernel based smoothing technique for any type of cortical data.

Previously cortical data have been smoothed by either solving diffusion equations [1, 3, 4] or the iterative applications of the first order heat kernel approximation [5]. However, the diffusion smoothing approaches require setting up a finite element scheme, which is computationally nontrivial, and mak- ing the algorithm converges. The iterative kernel smoothing method is simpler in comparison; however, since it is based on the repeated applications of the first order approximation, the convergence is very slow. To address the shortcomings of these methods, we propose a new technique that construct the heat kernel analytically using the spherical harmonics. The numerical implementation of the smoothing is validated against the known analytic solutions.

\section{SPHERICAL HARMONICS}

Consider the following parametrization of unit sphere $S^{2}$ :

$$
p=(x, y, z)=(\sin \theta \cos \varphi, \sin \theta \sin \varphi, \cos \theta)
$$

with $\theta \in[0, \pi], \varphi \in[0,2 \pi)$. The Laplacian corresponding to the parametrization is given by

$$
\Delta_{S^{2}}=\frac{1}{\sin \theta} \frac{\partial}{\partial \theta}\left(\sin \theta \frac{\partial}{\partial \theta}\right)+\frac{1}{\sin ^{2} \theta} \frac{\partial^{2}}{\partial^{2} \varphi} .
$$

For $f, h \in L^{2}\left(S^{2}\right)$, the space of square integrable functions in $S^{2}$, the inner product is defined as

$$
\langle f, h\rangle=\int_{S^{2}} f h d \mu=\int_{0}^{2 \pi} \int_{0}^{\pi} f(\theta, \varphi) h(\theta, \varphi) \sin \theta d \theta d \varphi,
$$

where $d \mu=\sin \theta d \theta d \varphi$ is the area element. The norm is defined as $\|f\|^{2}=\langle f, f\rangle$. Consider the following eigenequation $\Delta f+\lambda f=0$. There are $2 l+1$ eigenfunctions, denoted as $Y_{l m}(-l \leq m \leq l)$, corresponding to eigenvalue $\lambda=l(l+1)$. $Y_{l m}$ is called the spherical harmonic of degree $l$ and order $m$ [6]. The $2 l+1$ spherical harmonics of degree $l$ are

$$
Y_{l m}=\left\{\begin{array}{cc}
c_{l m} P_{l}^{|m|}(\cos \theta) \sin (|m| \varphi), & -l \leq m \leq-1, \\
\frac{c_{l m}}{\sqrt{2}} P_{l}^{0}(\cos \theta), & m=0, \\
c_{l m} P_{l}^{|m|}(\cos \theta) \cos (|m| \varphi), & 1 \leq m \leq l,
\end{array}\right.
$$

where $c_{l m}=\sqrt{\frac{2 l+1}{2 \pi} \frac{(l-|m|) !}{(l+|m|) !}}$ and $P_{l}^{m}$ is the associated Legendre polynomials of order $m$ given by

$$
P_{l}^{m}(x)=\frac{\left(1-x^{2}\right)^{m / 2}}{2^{l} l !} \frac{d^{l+m}}{d x^{l+m}}\left(x^{2}-1\right)^{l} .
$$




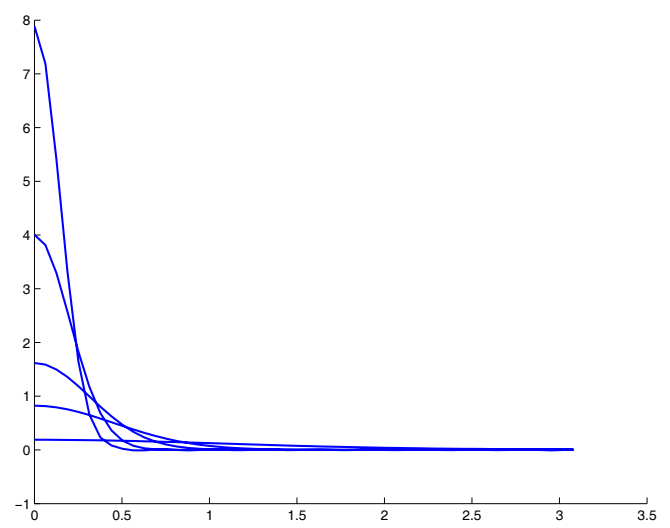

Fig. 1. Shape of the heat kernel with different bandwidth $\sigma=$ $0.01,0.02,0.05,0.1,0.5$ from top to bottom. The horizontal axis is the $\theta$ value from the north pole $(\theta=0)$ to the south pole $(\theta=\pi)$. As $\sigma$ becomes large, the heat kernel converges to constant value $1 / 4 \pi$.

Unlike other previous studies $[2,8,13]$ that use the complex numbered spherical harmonics, we are using a real function with different normalizing constants.

Any $f \in L^{2}\left(S^{2}\right)$ can be expressed as

$$
f=\sum_{l=0}^{\infty} \sum_{m=-l}^{l} \hat{f}_{l m} Y_{l m}
$$

where the Fourier coefficient $\hat{f}_{l m}=\left\langle f, Y_{l m}\right\rangle$. This can be viewed as the general Fourier expansion of the Hilbert space $L^{2}\left(S^{2}\right)$ on sphere. If we use up to the $d$-th degree spherical harmonics for modeling the cortical data, we need total $(2$. $0+1)+\cdots+(2 \cdot d+1)=(d+1)^{2}$ number of basis functions.

\section{HEAT KERNEL}

By extending Mercer's theorem [6] to $L^{2}\left(S^{2}\right)$, any continuous symmetric non-negative definite kernel $K(p, q)$ can be written as

$$
K(p, q)=\sum_{l=0}^{\infty} \sum_{m=-l}^{l} c_{l m} Y_{l m}(p) Y_{l m}(q)
$$

for some constants $c_{l m}$. The Green's function of the isotropic diffusion equation

$$
\frac{\partial f}{\partial t}=\Delta f
$$

in $S^{2}$ is called the heat kernel [5] and it can be written in the Mercer's theorem with $c_{l m}=e^{-l(l+1) \sigma}$. Then the heat kernel is given by

$$
K_{\sigma}(p, q)=\sum_{l=0}^{\infty}\left[e^{-l(l+1) \sigma} \sum_{m=-l}^{l} Y_{l m}(p) Y_{l m}(q)\right]
$$

with $\sigma=\sqrt{2 t}$. It directly generalize the Gaussian kernel in the Euclidean space to $S^{2}$ [5]. The kernel can be also seen as the transition probability of the isotropic diffusion process in $S^{2}$ and it should be symmetric along the geodesic circle centered around $p$, defined by $\mathcal{C}_{p}=\{q: p \cdot q=$ const. $\}$, where we used the vector product - in the Cartesian coordinate system. This implies that the heat kernel is isotropic with respect to $p$. Hence, if $p \cdot q=p \cdot r$, we have $K_{\sigma}(p, q)=$ $K_{\sigma}(p, r)$. This property can be used to simplify the expansion (3). Let us fix $\varphi=0$ and $p$ be the north pole, i.e. $p=$ $(0,0,1)$. Now by varying $q=(\sin \theta, 0, \cos \theta)$ for $0 \leq \theta=$ $\cos ^{-1}(p \cdot q) \leq \pi$, we have $Y_{l m}=0$ if $m \neq 0$. Then the inner summation in (3) can be written as

\section{Theorem. 1.}

$$
\sum_{m=-l}^{l} Y_{l m}(p) Y_{l m}(q)=\frac{2 l+1}{4 \pi} P_{l}^{0}(\cos \theta) .
$$

This implies that the sum of products of the spherical harmonics is a function of the angle between $p$ and $q$ only. For any $p$ other than the north pole, the left hand side is also a function of the angle between $p$ and $q$. The relationship (4) is usually called the harmonic addition theorem [9]. Then we have the following theorem:

Theorem. 2. For any $p$ and $q$ in $S^{2}$,

$$
K_{\sigma}(p, q)=\sum_{l=0}^{\infty} \frac{2 l+1}{4 \pi} e^{-l(l+1) \sigma} P_{l}^{0}(p \cdot q) .
$$

The same result is given in [2] based on more complicated proof. Our formulation and proof is more straightforward. Based on formulation (5), the shape of heat kernel is plotted in Figure 1 with varying bandwidths $\sigma=0.01,0.02,0.05$, $0.10,0.50$. As $\sigma$ increases, the kernel flattens out to a constant value, i.e. $\lim _{\sigma \rightarrow \infty} K_{\sigma}(p, q)=\frac{1}{4 \pi}$.

The full width at the half maximum (FWHM) of kernel has been widely used as a unit for measuring the amount of Gaussian kernel smoothing. For the usual 2D Gaussian kernel of the form $\frac{1}{2 \pi \sigma^{2}} e^{-\frac{x^{2}+y^{2}}{2 \sigma^{2}}}$, it is given by FWHM $=\sqrt{8 \ln 2} \sigma$. For heat kernel defined in $S^{2}$, since we can not find FWHM analytically in a close form, we estimate it numerically. The maximum of the heat kernel is obtained when $\theta=0$ in (5). Note $P_{l}^{0}(1)=1$ for all $l$. Then we solve numerically for $\theta$ in

$$
\sum_{l=0}^{d} \frac{2 l+1}{4 \pi} e^{-l(l+1) \sigma} P_{l}^{0}(\cos \theta)=\frac{1}{2} \sum_{l=0}^{d} \frac{2 l+1}{4 \pi} e^{-l(l+1) \sigma} .
$$

The FWHM for the heat kernel is then $2 \theta$. We used $d=20$ degree spherical harmonics in estimating the FWHM (Figure 2).

\section{HEAT KERNEL SMOOTHING}

The solution to the diffusion equation (2) with initial functional measurement $h(p)$ at time $t=\sigma^{2} / 2$ is given by the 


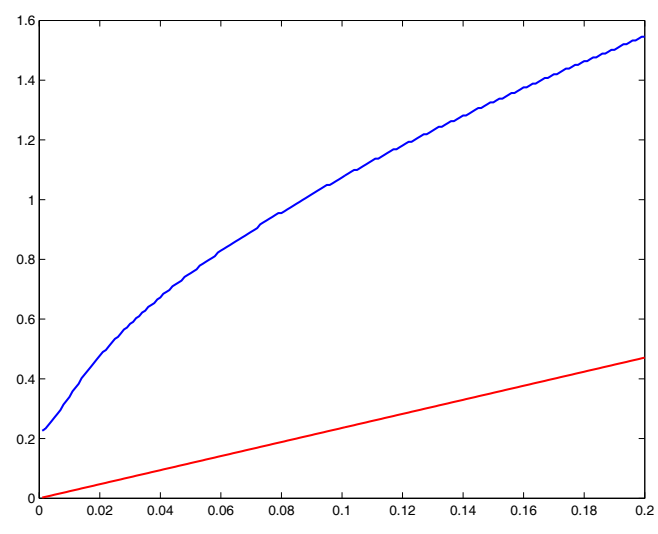

Fig. 2. Plot of FWHM (vertical) vs. bandwidth $\sigma$ (horizontal). The blue line is for the heat kernel and red line is for the isotropic Gaussian kernel in $\mathbb{R}^{2}$.

convolution:

$$
\begin{aligned}
K_{\sigma} * h(p) & =\int_{S^{2}} K_{\sigma}(p, q) h(q) d \mu(q) \\
& =\sum_{l=0}^{\infty} w_{j} \mathcal{P}_{l}(p)
\end{aligned}
$$

where the weighting factor $w_{l}=(2 l+1) e^{-l(l+1) \sigma}$ and integral

$$
\mathcal{P}_{l}(q)=\frac{1}{4 \pi} \int_{S^{2}} P_{l}^{0}(p \cdot q) h(q) d \mu(q) .
$$

The heat kernel smoothing can be given a geometric interpretation in the following way. Since the associated Legendre polynomial $P_{l}^{0}$ for the first two terms are $P_{0}^{0}\left(p \cdot q_{k}\right)=1$ and $P_{1}^{0}\left(p \cdot q_{k}\right)=p \cdot q_{k}$, we can see that the first term

$$
\mathcal{P}_{0}(p)=\frac{1}{4 \pi} \int_{S^{2}} h(q) d \mu(q)
$$

measures the average signal over the unit sphere while the second term

$$
\mathcal{P}_{1}(p)=\frac{1}{4 \pi} \int_{S^{2}} p \cdot q h(q) d \mu(q)
$$

measures the first moment of the signal with respect to $p$. The higher order terms of $\mathcal{P}_{l}(p)$ can be computed using the recurrence relation for the associated Legendre polynomial

$$
P_{l+1}^{0}(p \cdot q)=\frac{2 l+1}{l+1} p \cdot q P_{l}^{0}(p \cdot q)-\frac{l}{l+1} P_{l-1}^{0}(p \cdot q)
$$

so $P_{l}^{0}(p \cdot q)$ is the $l$-th degree polynomial of $p \cdot q$. Hence $\mathcal{P}_{l}(p)$ measures a signal quantity involving higher order moments.

\section{NUMERICAL IMPLEMENTATION}

The $S^{2}$ surface is realized as a mesh with 81,920 triangles. It is constructed from the deformable surface algorithm that

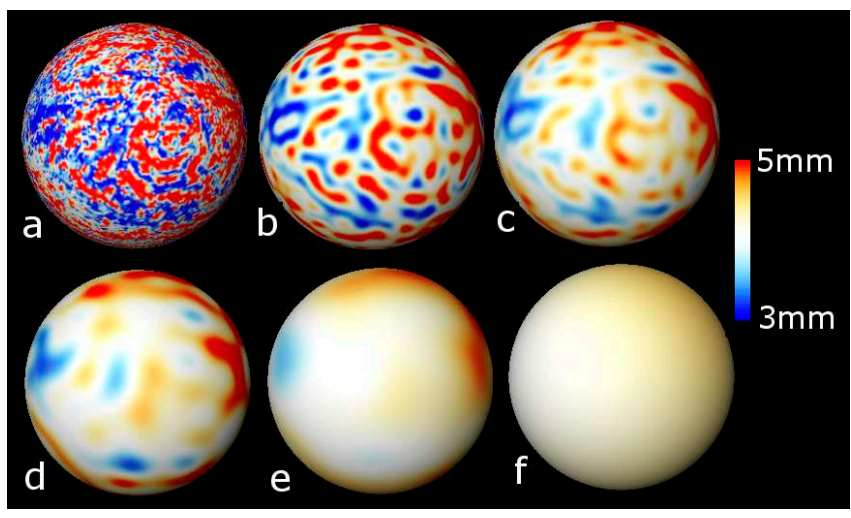

Fig. 3. Heat kernel smoothing on cortical thickness. (a) original cortical thickness data mapped onto a unit sphere, $(b, c)$ smoothing with $d=40$, and $\sigma=0.0001,0.001$ respectively. (d, e, f) smoothing with $d=20$, and $\sigma=0.001,0.01,0.1$ respectively.

gives a direct homological map from the human cortical surface to $S^{2}[4,11]$. Integral of form $\int_{S^{2}} f(q) d \mu(q)$ is approximated as a limit of the Riemann sum over triangle elements following the framework of finite element method (FEM) [4]. Let $n=40,962$ be the total number of nodes in the mesh. Suppose that triangular elements $T_{k_{1}}, \cdots, T_{k_{m}}$ are adjacent to each other at given node $q_{k}(1 \leq k \leq n)$. Then the total area of the mesh is computed as

$$
\int_{S^{2}} d \mu \doteq \frac{1}{3} \sum_{k=1}^{n} \sum_{i=1}^{k_{m}}\left|T_{k_{i}}\right|=12.565
$$

while the area of the unit sphere is $4 \pi=12.566$, the difference of less than $0.0001 \%$. So our triangular mesh is sufficiently fine enough to realize the $S^{2}$ surface accurately. The accuracy of our heat kernel smoothing is only restricted to the mesh resolution and the Riemann sum approximation should converges to the integral as the mesh resolution decreases.

In our numerical implementation, we avoided using formulation (7) due to the fact that the integral kernel $P_{l}^{0}(p \cdot q)$ is not separable. In this case, the running time is $O\left(n^{2}\right)$ for smoothing on every nodes in the mesh. On the other hand, if we use the form of the separable kernel (3), the smoothing can be done in $O(n)$. So our $O(n)$ implementation is to use the form

$$
K_{\sigma} * h(p)=\sum_{l=0}^{d} \sum_{m=-l}^{l} e^{-l(l+1) \sigma} Y_{l m}(p) \int_{S^{2}} Y_{l m}(q) h(q) d \mu(q) .
$$

The integral is approximated as

$$
\int_{S^{2}} f(q) d \mu(q) \doteq \frac{1}{3} \sum_{k=1}^{n} \sum_{i=1}^{k_{m}} f\left(q_{k}\right)\left|T_{k_{i}}\right|
$$




\begin{tabular}{|l|l|l|l|}
\hline$l^{\prime}$ & $m^{\prime}$ & integral & kernel smoothing \\
\hline 1 & 1 & 0.9999 & $-3.8854 \cdot 10^{-17}$ \\
10 & 5 & 0.9999 & $-3.7597 \cdot 10^{-17}$ \\
10 & 7 & 0.9999 & $4.9109 \cdot 10^{-17}$ \\
15 & 10 & 0.9998 & $-4.0601 \cdot 10^{-8}$ \\
20 & 4 & 1.0001 & $9.7029 \cdot 10^{-5}$ \\
20 & 10 & 0.9999 & $1.6212 \cdot 10^{-4}$ \\
20 & 20 & 0.9999 & $-1.1174 \cdot 10^{-4}$ \\
\hline
\end{tabular}

Table 1. Validation of heat kernel smoothing with test function $Y_{l^{\prime} m^{\prime}}$.

For an illustration, we mapped the cortical thickness data [5] obtained from MRI onto a unit sphere. Then we performed the smoothing with various bandwidths $\sigma$ and $d$ (Figure 3).

The numerical implementation is validated against analytical solutions. Let $h=e^{l^{\prime}\left(l^{\prime}+1\right)} Y_{l^{\prime} m^{\prime}}$ be an analytic test function. Then $K_{\sigma} * h(p)$ can be written as

$$
\begin{aligned}
& e^{l^{\prime}\left(l^{\prime}+1\right)} \sum_{l=0}^{\infty} \sum_{m=-l}^{l} e^{-l(l+1) \sigma} Y_{l m}(p) \int_{S^{2}} Y_{l m}(q) Y_{l^{\prime} m^{\prime}}(q) d \mu(q) \\
& =e^{l^{\prime}\left(l^{\prime}+1\right)} \sum_{l=0}^{\infty} \sum_{m=-l}^{l} e^{-l(l+1) \sigma} Y_{l m}(p) \delta_{l l^{\prime}} \delta_{m m^{\prime}}=Y_{l^{\prime} m^{\prime}}(p),
\end{aligned}
$$

where $\delta_{l l^{\prime}}$ is the Kroneker's delta. This exact analytical form gives a criteria for estimating the accuracy of our numerical method. The analytical solution is compared with the numerical approximation. The table 1 shows the result for selective $l^{\prime}$ and $m^{\prime}$ with $\sigma=0.01$ and $d=20$. The third column shows the numerical computation of integral

$$
\int_{S^{2}} Y_{l^{\prime} m^{\prime}}^{2}(p) d p=1
$$

showing the accuracy up to 3 decimal places. The fourth column shows the average difference between the numerical approximation and the expected theoretical value $Y_{l^{\prime} m^{\prime}}$.

\section{CONCLUSIONS}

We have developed a mathematical framework for performing kernel smoothing in a unit sphere. The heat kernel was constructed analytically using the spherical harmonics. Based on the FWHM vs $\sigma$ plot in Figure 2, we conclude that the heat kernel flattens out more rapidly than the corresponding Gaussian kernel. Based on the exact mathematical formulation, we developed the $O(n)$ smoothing algorithm. The algorithm is validated against the analytical solution.

\section{REFERENCES}

[1] A. Andrade, Kherif, J. F., Mangin, K.J. Worsley, A. Paradis, O. Simon, S. Dehaene, D. Le Bihan, and J-B. Po- line, "Detection of fmri activation using cortical surface mapping," Human Brain Mapping, vol. 12, pp. 79-93, 2001.

[2] T. Bulow, "Spherical diffusion for 3d surface smoothing," IEEE Transactions on Pattern Analysis and Machine Intelligence, vol. 26, pp. 1650-1654, 2004.

[3] A. Cachia, J.-F. Mangin, Riviére D., D. PapadopoulosOrfanos, F. Kherif, I. Bloch, and J. Régis, "A generic framework for parcellation of the cortical surface into gyri using geodesic voronoï diagrams," Image Analysis, vol. 7, pp. 403-416, 2003.

[4] M.K. Chung, K.J. Worsley, S. Robbins, and A.C. Evans, "Tensor-based brain surface modeling and analysis," in IEEE Conference on Computer Vision and Pattern Recognition (CVPR), 2003, vol. I, pp. 467-473.

[5] M.K. Chung, S. Robbins, A.C. Evans, "Unified statistical approach to cortical thickness analysis," Information Processing in Medical Imaging (IPMI), in Lecture Notes in Computer Science (LNCS) 3565:627-638. SpringerVerlag. 2005.

[6] R. Courant and D. Hilbert., Methods of Mathematical Physics, Volume 1., Interscience, New York, 1953.

[7] A.M. Dale and B. Fischl, "Cortical surface-based analysis i. segmentation and surface reconstruction," $\mathrm{Neu}$ roImage, vol. 9, pp. 179-194, 1999.

[8] M.S. Gudio Gerig and G. Szekely, "Statistical shape models for segmentation and structural analysis," in Proceedings of IEEE International Symposium on Biomedical Imaging (ISBI), 2004.

[9] Groemer, H., Geometric Applications of Fourier Series and Spherical Harmonics, Cambridge University Press, 1996.

[10] M.K., Hurdal, P.L., Bowers, et al. "Quasi-conformally flat mapping the human cerebellum," in Medical Image Computing and Computer-Assisted Intervention (MICCAI), 1999.

[11] J.D. MacDonald, N. Kabani, D. Avis, and A.C. Evans, "Automated 3-d extraction of inner and outer surfaces of cerebral cortex from mri," Neurolmage, vol. 12, pp. 340-356, 2000.

[12] S. Rosenberg, The Laplacian on a Riemannian Manifold, Cambridge University Press, 1997.

[13] Shen, L., Ford, J., Makedon, F., Saykin, A. A surfacebased approach for classification of 3D neuroanatomical structures. Intelligent Data Analysis, 8(5). 2004. 